\title{
Compte rendu de Making the Modern Primitive de Michelle MacCarthy
}

\section{Aurélie Condevaux}

\section{OpenEdition}

1 Journals

\section{Édition électronique}

URL : http://journals.openedition.org/jso/7857

DOI : $10.4000 /$ jso.7857

ISSN : 1760-7256

\section{Éditeur}

Société des océanistes

\section{Édition imprimée}

Date de publication : 15 décembre 2017

Pagination : 356-358

ISSN : 0300-953x

\section{Référence électronique}

Aurélie Condevaux, "Compte rendu de Making the Modern Primitive de Michelle MacCarthy », Journal de la Société des Océanistes [En ligne], 144-145 | 2017, mis en ligne le 15 décembre 2017, consulté le 15 mars 2021. URL : http://journals.openedition.org/jso/7857 ; DOI : https://doi.org/10.4000/jso.7857

Ce document a été généré automatiquement le 15 mars 2021.

\section{(†)

Journal de la société des océanistes est mis à disposition selon les termes de la Licence Creative Commons Attribution - Pas d'Utilisation Commerciale - Pas de Modification 4.0 International. 


\title{
Compte rendu de Making the Modern Primitive de Michelle MacCarthy
}

\author{
Aurélie Condevaux
}

\section{RÉFÉRENCE}

MACCARTHY Michelle, 2017. Making the Modern Primitive. Cultural Tourism in the Trobriand Islands, Honolulu, University of Hawai'i Press, 304 p., glossaire, bibliogr., index, 25 ill. N\&B.

1 Les recherches en sciences sociales sur le tourisme examinent aujourd'hui l'idée selon laquelle nous serions entrés dans une nouvelle ère touristique, parfois qualifiée de " post-tourisme ", caractérisée notamment par un affaiblissement des frontières entre l'« ailleurs» et l'« ici », entre l'« exotique » et le "quotidien», entre " ordinaire » et « extraordinaire » (cf. notamment Bourdeau, 2012) et par un rapport plus distancié à la question de l'authenticité. L'ouvrage de Michelle MacCarthy, Making the Modern Primitive. Cultural Tourism in the Trobriand Islands, vient rappeler que ce schéma ne peut être appliqué uniformément pour penser les dynamiques dans le champ touristique, la recherche de l'« exotique », de l'« extraordinaire » et de l'« authentique » étant encore au cœur des motivations de certains touristes.

2 Certes le caractère exceptionnel du lieu d'enquête choisi, l'île de Kiriwina dans l'archipel des Trobriand, implique en soi de s'intéresser à des formes bien particulières de tourisme. Une île fréquentée par 186 touristes sur une période de dix-huit mois (durée du premier séjour de l'auteure à Kiriwina) peut à première vue ne pas sembler être le lieu idéal pour entreprendre une ethnographie des interactions touristiques; c'est pourtant une entreprise qui se révèle extrêmement riche d'enseignements, la portée de ces derniers allant bien au-delà du cas des espaces peu touristiques. L'enquête de terrain de longue durée conduite en 2009 et 2010 permet à Michelle MacCarthy de se revendiquer, dans ce contexte, d'une certaine exhaustivité du regard (elle a interviewé près de la moitié des touristes qui se sont rendus sur l'île durant son 
séjour). Elle offre en outre un nouvel exemple de l'apport spécifique de la discipline à l'étude du tourisme, l'ethnographie permettant de saisir les "deux côtés » de la rencontre touristique, l'enquêteuse devenant, avec le temps, "ni tout à fait touriste ", ni «tout à fait trobriandaise ", selon son expression. Le caractère non ordinaire de ce terrain vient également du fait que la fréquentation touristique y est plus faible en 2009 qu'elle ne l'était au début des années 1970, lorsqu'Annette Weiner y effectua ses premiers séjours de recherche, Kiriwina étant alors un lieu d'excursion prisé pour les Australiens résidant à Port Moresby. Enfin, ce terrain est loin d'être ordinaire dans la mesure où il est indissociable du nom de l'un des «pères fondateurs » de la discipline, B. Malinowski. L'importance de cette figure est omniprésente dans l'ouvrage, non seulement pour l'anthropologue, mais aussi pour les touristes et les Trobriandais. Le premier chapitre offre un regard réflexif sur les conditions d'enquête dans ce contexte particulier. Michelle MacCarthy ne décrit pas seulement le poids de cette lignée de prédécesseurs illustres sur sa propre recherche, elle décrit aussi avec humour les relations d'aide mais aussi d'évitement qui peuvent exister entre anthropologues d'une même génération.

3 Ces différentes caractéristiques - faiblesse de la fréquentation touristique et difficulté d'accès, iconicité du lieu dans la littérature anthropologique - font des îles Trobriand une destination idéale pour des touristes en quête d'une culture "primitive». Les citations des nombreux entretiens que l'auteure a conduits avec les visiteurs nous convainc que l'opposition entre " primitif » et « moderne » est bien au cœur du métadiscours qui sert de cadre de référence à ces touristes, et bien que ces derniers ne constituent pas un groupe homogène. Leur profil est dévoilé au fur et à mesure de l'ouvrage.

côté des touristes voyageant en groupes organisés, notamment en croisières, etrestant sur l'île pour un temps très court (ces voyages sont organisés notamment au départ de Port Moresby et d'Australie, mais aussi d'Italie), d'autres, indépendants, revendiquent un esprit d'aventure : anciens étudiants en anthropologie, traders reconvertis à des modes de vie plus alternatifs, ou simples backpackers. La centaine d'entretiens conduits avec ces visiteurs est complétée par la familiarisation avec la vie sur Kiriwina, permise par un séjour de longue durée dans une famille du village de Modawosi, et l'observation de nombreuses situations d'interaction.

4 Constitué de huit chapitres, l'ouvrage revient sur des thèmes centraux dans l'anthropologie du tourisme: les images et les imaginaires, les performances de musique et de danse, la production et la vente de souvenirs. L'auteure identifie ainsi quatre principaux lieux de la rencontre et de l'échange interculturel : les performances culturelles et les festivals, les visites de village, l'achat de souvenirs, et l'acte de photographier. C'est à travers ces quatre principaux champs de l'interaction que les acteurs construisent et manipulent les concepts de "tradition », de "culture » et d'" authenticité ». Le travail mené sur les imaginaires permet en partie de comprendre comment le «méta-discours» du primitivisme perdure au $\mathrm{xxI}^{\mathrm{e}}$ siècle. La spécificité dans le cas trobriandais vient du fait que la mosaïque de représentations visuelles et discursives construites autour de cette destination doit beaucoup, à côté de la littérature, du cinéma et des romans, aux travaux anthropologiques et à la manière dont ceux-ci sont commentés dans d'autres supports médiatiques : Malinowski est en effet une référence pour de nombreux touristes venant visiter les îles Trobriand, mais aussi pour les Trobriandais eux-mêmes. L'auteure insiste également sur la réciprocité des regards et des imaginaires. Le chapitre sur l'achat de souvenirs revient sur les 
discussions ouvertes par N. Graburn (1976) dans les années 1970 à ce sujet. L'auteure examine à la fois la question du statut de l'objet (souvenir/art/artisanat, mais aussi marchandise/don) et les facteurs expliquant la création de valeur autour de ces productions matérielles dans des contextes interculturels. S'il me semble que ce chapitre vient surtout confirmer des éléments déjà largement acceptés (qu'un objet peut avoir plusieurs statuts en même temps), il apporte des éléments ethnographiques précis sur les critères utilisés par les touristes pour distinguer l'authentique de l'inauthentique. C'est également par le biais d'une ethnographie fine que l'auteure aborde la question de la négociation des images dans l'interaction touristique et des performances culturelles.

Outre la contribution de l'auteure à la réflexion sur ces diverses thématiques centrales dans l'étude du tourisme, l'apport principal de l'ouvrage me semble résider dans la réflexion proposée sur la question de la marchandisation ou commercialisation. Alors que la monétarisation de l'économie était évoquée dans les premiers travaux anthropologiques sur le tourisme comme un « risque " pour la reproduction culturelle et sociale, la contribution de M. McCarthy est exemplaire d'un renouvellement de l'approche sur ce sujet. Le tourisme devient un contexte ou un phénomène parmi d'autres (on peut évoquer les ventes aux enchères par exemple [Geismar, 2013]) qui permet d'examiner les rencontres entre des univers où les normes dominantes de l'échange, et les critères mobilisés pour attribuer une valeur à un objet ou à un élément non matériel, diffèrent. Les questions soulevées par C.Gregory en 1982 sur les différences entre économie de don et économie de marché sont posées ici à nouveaux frais. Si le thème sert de fil conducteur à l'ouvrage dans son ensemble, c'est dans le deuxième chapitre - qui pose le cadre théorique - et dans le dernier "Cashing in on Culture » que l'on trouvera les éléments les plus importants sur ces questions. Non seulement l'auteure montre comment les objets peuvent être à la fois des marchandises et des dons et circulent entre différentes sphères d'échange, mais aussi que la " marchandise » n'est pas nécessairement dépersonnalisée. Ce point en particulier amène une réflexion sur la question de l'expérience. L'auteure montre l'ambiguïté qui est au cœur de la commercialisation de cette "marchandise " particulière. Le touriste paye non seulement pour des prestations (hébergement, nourriture etc.), pour des objets, ainsi que pour des performances visuelles et éphémères, mais aussi pour l'expérience en elle-même, élément éminemment unique, qui n'est ni reproductible ni tout à fait " aliénable » dans la mesure où ce qui en fait la valeur, ce sont précisément les relations et leur personnalisation - qui entourent la transaction. Ainsi, l'auteure conclut que l'expérience touristique est une marchandise d'un type particulier, dans laquelle la distance sociale et l'attente de réciprocité sont manipulées, afin de jeter un voile sur les dimensions marchandes de l'échange. Les conclusions de l'ouvrage trouveront un écho certain dans les recherches actuelles sur le tourisme, où cette notion d'expérience a gagné en importance, mais aussi dans les travaux, en Océanie et ailleurs, qui continuent d'interroger les liens entre échanges marchands et non marchands et les crit ères d'attribution de la valeur. 


\section{BIBLIOGRAPHIE}

BOURDEAU Philippe, 2012. Le tourisme réinventé par ses périphéries ?, in F. Bourlon, M. Osorio, P. Mao et T. Gale, Explorando las nuevas fronteras del turismo. Perspectivas de la invetigacion en turismo, Nire Negro, pp. 31-48.

GEISMAR Haidy, 2013. Treasured possessions. Indigenous Interventions into Cultural and Intellectual Property, Durham et Londres, Duke University Press.

GRABURN Nelson H.H. (ed.), 1976. Ethnic and Tourist Arts: Cultural Expressions from the Fourth World, Berkeley, University of California Press.

GREGORY, Christopher, 1982. Gifts and commodities, Londres, Academic Press.

\section{AUTEUR}

\section{AURÉLIE CONDEVAUX}

EIREST-Université Paris 1 Panthéon-Sorbonne 